\title{
Pre-radiotherapy FDG PET predicts radiation pneumonitis in lung cancer
}

\author{
Richard Castillo ${ }^{2}$, Ngoc Pham ${ }^{1}$, Sobiya Ansari ${ }^{4,6}$, Dmitriy Meshkov ${ }^{1}$, Sarah Castillo ${ }^{4}$, Min Li $^{4}$, Adenike Olanrewaju $^{4}$, \\ Brian Hobbs ${ }^{3}$, Edward Castillo ${ }^{4,5}$ and Thomas Guerrero ${ }^{1,4,5,7^{*}}$
}

\begin{abstract}
Background: A retrospective analysis is performed to determine if pre-treatment $\left[{ }^{18} \mathrm{~F}\right]-2$-fluoro-2-deoxyglucose positron emission tomography/computed tomography (FDG PET/CT) image derived parameters can predict radiation pneumonitis (RP) clinical symptoms in lung cancer patients.

Methods and Materials: We retrospectively studied 100 non-small cell lung cancer (NSCLC) patients who underwent FDG PET/CT imaging before initiation of radiotherapy (RT). Pneumonitis symptoms were evaluated using the Common Terminology Criteria for Adverse Events version 4.0 (CTCAEv4) from the consensus of 5 clinicians. Using the cumulative distribution of pre-treatment standard uptake values (SUV) within the lungs, the 80th to 95th percentile SUV values $\left(S_{U} V_{80}\right.$ to $\left.S_{U V} V_{95}\right)$ were determined. The effect of pre-RT FDG uptake, dose, patient and treatment characteristics on pulmonary toxicity was studied using multiple logistic regression.

Results: The study subjects were treated with 3D conformal RT ( $n=23)$, intensity modulated RT $(n=64)$, and proton therapy $(n=13)$. Multiple logistic regression analysis demonstrated that elevated pre-RT lung FDG uptake on staging FDG PET was related to development of RP symptoms after RT. A patient of average age and $V_{30}$ with $\mathrm{SUV}_{95}=1.5$ was an estimated 6.9 times more likely to develop grade $\geq 2$ radiation pneumonitis when compared to a patient with $S U V_{95}=0.5$ of the same age and identical $V_{30}$. Receiver operating characteristic curve analysis showed the area under the curve was $0.78(95 \% \mathrm{Cl}=0.69-0.87)$. The $\mathrm{CT}$ imaging and dosimetry parameters were found to be poor predictors of RP symptoms.
\end{abstract}

Conclusions: The pretreatment pulmonary FDG uptake, as quantified by the $S U V_{95}$, predicted symptoms of RP in this study. Elevation in this pre-treatment biomarker identifies a patient group at high risk for post-treatment symptomatic RP.

Keywords: Standard uptake value, PET/CT, Radiation pneumonitis, NSCLC, Thoracic radiotherapy, Imaging biomarker

\section{Introductions}

Radiation pneumonitis (RP), an inflammatory reaction within lung tissue secondary to radiation damage [1,2], is a severe and potentially fatal complication of thoracic radiotherapy (RT). Symptoms of RP include dyspnea, non-productive cough, shortness of breath, fever, and changes in pulmonary function. RP-associated mortality has been noted in the treatment of many cancers including breast [3], esophageal $[4,5]$, lung [6,7], and mesothelioma

\footnotetext{
* Correspondence: tguerrero@mdanderson.org

'The University of Texas Health Science Center, Houston, TX, USA

${ }^{4}$ Radiation Oncology, The University of Texas MD Anderson Cancer Center, Houston, TX, USA

Full list of author information is available at the end of the article
}

[8-10]. Furthermore, the mortality rate among non-small cell lung cancer (NSCLC) patients experiencing severe $\mathrm{RP}$ symptoms requiring hospitalization approaches $50 \%$ [11]. The variability of RP symptoms onset and intensity with respect to patient specific radiation dose, irradiated lung volume, and pulmonary function has made past prognostication efforts futile [12]. Treatment toxicity including RP remains a barrier to radiation dose escalation in lung cancer [13]. Because RP plays such an important role in defining the therapeutic index for lung cancer, clearly there remains a significant need for patient specific prognostication.

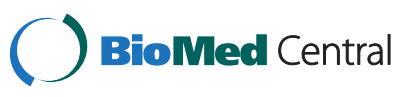

(c) 2014 Castillo et al.; licensee BioMed Central Ltd. This is an Open Access article distributed under the terms of the Creative Commons Attribution License (http://creativecommons.org/licenses/by/2.0), which permits unrestricted use, distribution, and reproduction in any medium, provided the original work is properly credited. The Creative Commons Public Domain Dedication waiver (http://creativecommons.org/publicdomain/zero/1.0/) applies to the data made available in this article, unless otherwise stated. 
Numerous factors such as percentage of lung irradiated [14-16] and chemotherapy type $[3,7,17]$ have been shown to affect occurrence and degree of RP. Another such factor, interstitial pneumonitis (IP) on pretreatment computed tomography (CT) scans, has been shown to predict an increased risk of symptomatic RP [18-20]. Makimoto et al. [18] found that in patients with primary lung cancer, pre-existing lung disease evidenced by pretreatment radiographic changes was associated with a higher incidence of RP $(47.1 \%$ vs. $5.3 \%, \mathrm{p}<0.001)$. Another study showed a correlation between severe RP and pretreatment IP foci in the lung periphery on $\mathrm{CT}$, although exclusion of patients with IP from receiving SBRT led to a reduction in the incidence of severe RP from $18.8 \%$ to $3.5 \%(\mathrm{p}=0.042)$ in subsequent cases [20]. Additionally, among 106 patients treated with thoracic RT, pretreatment interstitial changes on CT were associated with a higher incidence of grade $\geq 3$ RP $(26 \%$ versus $3 \%, p<0.001)$ [19]. CT scans and $x$-rays are not the only method to detect pulmonary inflammatory processes. With $\left[{ }^{18} \mathrm{~F}\right]$-2-fluoro-2-deoxyglucose positron emission tomography (FDG PET) imaging, pulmonary inflammation manifests as enhanced FDG uptake, thereby allowing for the quantitative assessment of pneumonitis [21-23]. Recently, Petit et al. [24] performed a retrospective study of 101 NSCLC patients to evaluate the correlation between symptomatic RP and pre-RT FDG PET/CT evidence of pulmonary inflammation. They report that the 95th percentile of the standard uptake value $\left(\mathrm{SUV}_{95}\right)$ within the lungs was predictive of RP on multivariate analysis $(p=0.016)$, suggesting that the $\mathrm{SUV}_{95}$ can be used to screen for RP risk during thoracic RT treatment planning [24].

In this retrospective study, pre-RT FDG PET/CT image derived factors are analyzed as potential prognostic biomarkers of symptomatic RP in NSCLC patients, testing the findings reported by Petit et al. [24]. We hypothesize that these pre-RT image derived factors identify individuals at high risk for symptomatic RP.

\section{Methods and Materials Patient population}

The study population consisted of 100 non-small cell lung cancer patients who were treated in the Department of Radiation Oncology at the University of Texas M. D. Anderson Cancer Center between July 2004 and May 2012, and who had their staging PET/CT imaging within 90 days prior to the start of radiotherapy. All study subjects had biopsy-proven NSCLC, and their imaging studies are available in the electronic medical records. Patient characteristics were obtained for each study subject including age, sex, disease stage, tumor location, smoking history, tumor histologic type, radiation planning, interval between staging PET and RT, concurrent chemotherapy, and pre-existing lung disease (as assessed by FEV1 and
DLCO parameters). Patient identifiers were removed in accordance with a retrospective study protocol (PA11-0801) approved by the MD Anderson Institutional Review Board. Waiver of informed consent was approved by the Institutional Review Board for this retrospective study protocol.

\section{${ }^{18}$ F-FDG PET/CT imaging}

Patients fasted 6 hours prior to the ${ }^{18} \mathrm{~F}$-FDG PET/CT imaging session and were required to have blood glucose levels $<120 \mathrm{mg} / \mathrm{dL}$. Intravenous injection of 629 (range: 550 - 740) MBq of ${ }^{18}$ F-FDG occurred 60 (range: 52-110) minutes prior the image acquisition. The General Electric Discovery ST PET/CT scanner (GE Medical Systems, Waukesha, WI) was used to acquire the ${ }^{18}$ F-FDG PET/ $\mathrm{CT}$ images. Patients were instructed to breath normally during the PET emission acquisition. The ${ }^{18} \mathrm{~F}$-FDG PET images included in this study acquired before 2006 were attenuation corrected using a non-contrast mid-inspiratory breath-hold CT, and those after used a respiratory averaged CT [25]. PET/CT images were acquired from mid-thigh to the skull base with arms raised. Standard uptake values (SUV) were calculated from the attenuation corrected ${ }^{18} \mathrm{~F}$-FDG PET emission images using the following equation [26]:

$$
\text { Standard Uptake Value }=\frac{{ }^{18} F-F G D \text { count rate per } m L \times \text { body weight }(\mathrm{gm})}{\text { decay corrected }{ }^{18} F-F D G \text { injected dose }(B q)}
$$

\section{Radiation treatment planning}

Treatment planning for megavoltage $\mathrm{x}$-ray cases was performed using the Pinnacle ${ }^{3}$ version 7.6c or 8.0u treatmentplanning system (Philips Medical Systems, Andover, MA). Proton therapy cases were planned using the respiratory averaged CT and the Eclipse treatment planning system (Varian Medical Systems, Palo Alto, CA). Gross target delineation and margin generation were performed in a consistent manner, as previously reported by our group [27]. Radiation dose was calculated using either free-breathing treatment planning CT data (most cases) or averaged CT data obtained from the treatment planning 4D CT image set $[28,29]$. All treatment plans and field arrangements were prospectively reviewed in quality assurance meetings in which consensus was obtained according to each patient's clinical circumstances. The radiation dose distributions were all calculated using lung heterogeneity corrections. The mean lung dose (MLD) and the percentage of lung volume irradiated to above 5 Gy or CGE $\left(V_{5}\right), 10$ Gy or CGE $\left(\mathrm{V}_{10}\right), 20$ Gy or CGE $\left(\mathrm{V}_{20}\right)$, and 30 Gy or CGE $\left(\mathrm{V}_{30}\right)$ were used as dosimetric parameters to represent the lung volumes irradiated.

For proton cases, all plans were designed for passive scattering delivery. Using a constant relative biological 
effectiveness (RBE) of 1.1, proton therapy doses were converted to ${ }^{60} \mathrm{Co}$ Gray Equivalents (CGE).

\section{Clinical Toxicity and Radiation Parameters}

Pneumonitis was scored using the National Cancer Institute Common Terminology Criteria for Adverse Events version 4 (CTCAE v4). All patient documents were used in the scoring, including consultation notes, radiographic images, clinic notes, summaries and scanned outside medical records until 6 months after completing radiation. A simple group consensus of 5 clinicians was used for each score. Cases were reviewed until all discrepancies were resolved by unanimous agreement. Clinically symptomatic pneumonitis was defined as grade 2 or higher. All patients with RP scores $>1$ had radiographic findings consistent with RP within the radiotherapy treatment field. These findings were evident on follow-up CT imaging and/or $\mathrm{PET} / \mathrm{CT}$.

\section{Image analysis}

The treatment plan and PET/CT images for each patient were processed and evaluated using custom MATLAB software (v2011a, Mathworks, Inc.). Lung regions of interest (ROIs) were segmented semi-automatically using histogram segmentation of the lung parenchyma and removal of the central airway by connectivity. PET spill-over artifacts (Figure 1) attributable to liver, heart, or tumor activities were manually contoured for exclusion from the segmented lung volume. Attenuation cold-spot artifacts at the diaphragm surface [30] were also manually removed. The effect of manual editing on the lung ROI and subsequent analysis was assessed according to repeat image segmentation performed by 3 independent secondary reviewers in a subsample of 10 patients (10\% of all cases). The primary reader binary lung ROI was used in subsequent analyses.

\section{Pretreatment PET/CT analysis}

Using the pretreatment FDG PET images, the SUV of all voxels within the lung ROI were binned into histograms, and the mean SUV (SUV $\left.{ }_{\text {mean }}\right)$, the standard deviation of the SUV $\left(\mathrm{SUV}_{\mathrm{SD}}\right)$, and the maximum SUV $\left(\mathrm{SUV}_{\max }\right)$ were calculated as described in Petit et al. [24]. A cumulative probability distribution was constructed from each histogram (Figure 2) and used to determine the 80th, 90th, and 95th percentiles of the SUV distribution, hereafter designated: $\mathrm{SUV}_{80}, \mathrm{SUV}_{90}$, and $\mathrm{SUV}_{95}$, respectively. To determine if pre-treatment CT density could predict RP, the cumulative density parameters mentioned above were also calculated for Hounsfield Unit (HU) of the CT scan: the $\mathrm{HU}_{\text {mean }}, \mathrm{HU}_{\mathrm{SD}}, \mathrm{HU}_{\text {max }}, \mathrm{HU}_{80}, \mathrm{HU}_{90}$, and $\mathrm{HU}_{95}$.

\section{Statistical Analysis}

Categorical variables (i.e., gender, tumor stage, tumor location, tumor histologic type, radiotherapy modality, chemotherapy status, smoking status, GOLD classification) were summarized using frequency tables; evaluated for association with symptomatic (grade $\geq 2$ ) RP using Pearson's

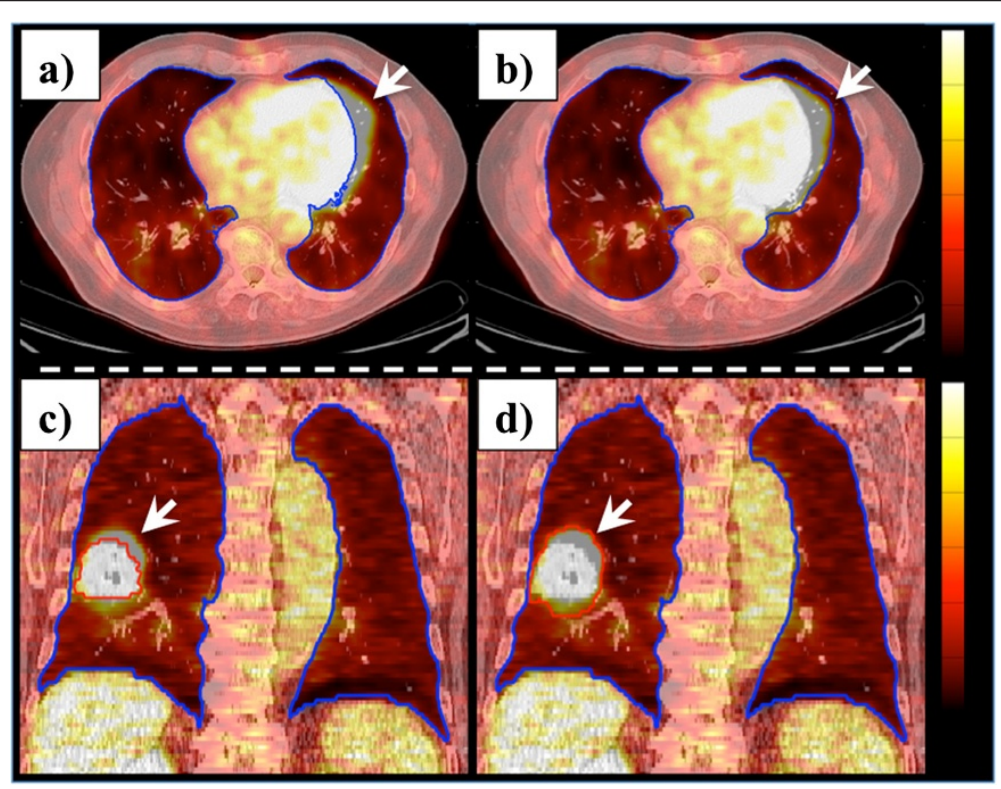

Figure 1 Lung segmentation and removal of PET spill-over activity artifacts. Semi-automated histogram segmentation and morphological region growing were used to delineate the set of lung voxels on the pre-treatment staging PET/CT studies. PET spill-over activities into the lung $\mathrm{ROI}$ were manually contoured for exclusion. Top row: lung ROI shown (a) before and (b) after manual correction of cardiac spill-over activity. Bottom row: the region of exclusion due to tumor is show in red (c) before and (d) after manual correction. 


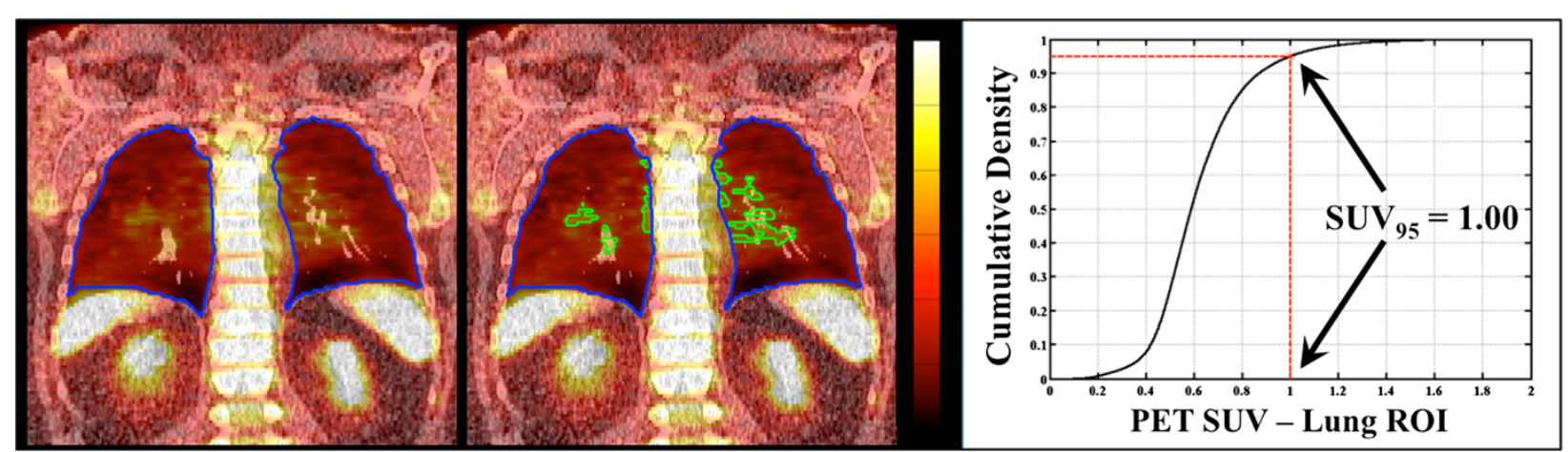

Figure 2 Quantifying the SUV $_{\mathbf{9 5}}$. SUV values within the lung are determined by semi-automated segmentation of the lung voxels from the pre-treatment staging PET/CT study (left). The cumulative distribution of SUV values is constructed from the voxel values within the lung ROI. The 80th, 90th, and 95th percentiles are obtained from these distributions to yield the corresponding SUV ${ }_{80}, S_{\text {SUV }}$, and SUV $V_{95}$. The SUV $V_{95}$ is depicted graphically (right) for the example case, with the ROI $\geq \mathrm{SUV}_{95}$ shown superimposed (middle).

chi-squared test for marginal homogeneity. Age and the interval between radiotherapy and PET imaging were summarized by median and range; evaluated for association with symptomatic (grade $\geq 2$ ) radiation pneumonitis using Mann-Whitney U tests. Univariate logistic regression analyses were used to predict symptomatic (grade $\geq 2$ ) RP as functions of pre-RT pulmonary and dosimetry characteristics (i.e., SUV, HU, MLD, irradiated volume, FEV1\%, DLCO\%). Post-hoc application of the sequentially rejective Bonferroni method [31] was used to adjust for multiplicity among the six SUV analyses.

Multiple logistic regression inference used stepwise backward model selection based on Akaike information criterion [32]. Results are provided for the best subset of predictors $\left(\mathrm{SUV}_{95}, \mathrm{~V}_{30}\right.$, age). Partial effects were evaluated for significance using two-sided Wald tests. Nagelkerke's coefficient of multiple determination [33] is used to report the proportion reduction in error variation obtained by incorporating the predictors. The resultant receiver operating characteristic (ROC) curve is provided with Delong's 95\% confidence interval [34] for the area under the curve (AUC) and Youden's optimal [35] specificity and sensitivity. Additionally, recursive partitioning analysis [36] was used to formulate a binary classification tree based upon both $\mathrm{SUV}_{95}$ and $\mathrm{V}_{30}$. Kaplan-Meier curves were used to compare time to radiation pneumonitis symptom development among the observed terciles of $\mathrm{SUV}_{95}\left(\mathrm{SUV}_{95}<0.99\right.$, $\left.0.99 \leq \mathrm{SUV}_{95}<1.2, \mathrm{SUV}_{95} \geq 1.2\right)$; Cox proportional hazard regression was used to evaluate the rate of RP symptom development as a function of $\mathrm{SUV}_{95}$ adjusted for patient and treatment characteristics. Stepwise backward model selection used generalized Akaike information criterion [29]. Results are provided for the best subset of predictors $\left(\mathrm{SUV}_{95}, \mathrm{~V}_{30}\right.$, age). Inter-reviewer variability in determination of $\mathrm{SUV}_{95}$ was assessed for 3 independent reviewers in a subsample of 10 patients; $95 \%$ limits of agreement were estimated using one-way mixed effects ANOVA [37].
The resultant Bland-Altman plot [38] is provided. All tests were two sided with $\alpha=0.05$ to confer statistical significance. All plots and analyses were performed using the statistical software R ( $\mathrm{R}$ Development Core Team, http://www.r-project.org) version 3.0.

\section{Results}

\section{Patient Characteristics and RP Symptoms}

An overview of the 100 study subjects and their characteristics is presented in Tables 1 and 2 . Of the study participants, 14 (14\%) were treated with RT alone while $86(86 \%)$ received concurrent chemo-radiation (chemoRT). The prescription dose range was 36 to 74 Gy (median 66) over 12-37 fractions (median 35). The mean lung dose was between 2.88 and $29.43 \mathrm{~Gy}$ (median 17.86 Gy). Consensus CTCAEv4 RP symptom scores were: 10 patients (10\%) had no evidence of respiratory symptoms or imaging changes (grade 0), 31 patients $(31 \%)$ had only radiographic or mild respiratory symptoms without requirement of intervention (grade 1), 27 patients (27\%) had post-RT respiratory symptoms affecting the extended activities of daily living (grade 2), 23 (23\%) required oxygen (grade 3), 1 (1\%) respiratory failure requiring intubation (grade 4 ) and, 8 (8\%) died from respiratory compromise (grade 5). A total of $60 \%$ of the patients experienced symptomatic RP.

The patient demographics, stage, tumor location, tumor histology, treatment type and smoking history are reported in Table 1 for the total and symptomatic (CTCAEv4 RP grade $\geq 2$ ). Treatment characteristics and outcomes are listed in Table 2. The data lacked significant evidence to conclude that the presence of symptomatic RT was associated with other clinical factors including tumor stage, histology, location, type of RT, or preexisting lung disease based on FEV1 parameters, as well as any CT-derived imaging parameters. 
Table 1 Patient Characteristics

\begin{tabular}{|c|c|c|c|c|c|}
\hline Parameter & Total (\%) & Symptomatic ${ }^{\mathrm{a}} \mathrm{N}(\%)$ & p-value & Treatment dose (Gy or CGE) & \\
\hline No of patients & $100(100)$ & $59(59)$ & & Range (median) & $36-74(66)$ \\
\hline Gender & & & 0.65 & Mean lung dose (Gy or CGE) & \\
\hline Male/Female & $60(60) / 40(40)$ & $35(58.3) / 26(65)$ & & Range (median) & $2.88-29.43(17.86)$ \\
\hline Age & & & 0.011 & ${ }^{a}$ Radiation pneumonitis symptom score, n (\%) & \\
\hline Symptomatic (IQR) & & $59.5-73$ yrs & & 0 & $10(10)$ \\
\hline Asymptomatic (IQR) & & $54-66$ yrs & & 1 & $31(31)$ \\
\hline Stage $^{b}$ & & & 0.36 & 2 & $27(27)$ \\
\hline 1 & $6(6)$ & $1(16.7)$ & & 3 & $23(23)$ \\
\hline$\|$ & $5(5)$ & $4(80)$ & & 4 & $1(1)$ \\
\hline III & $78(78)$ & $48(61.5)$ & & 5 & $8(8)$ \\
\hline IV & $11(11)$ & $6(54.5)$ & & Pre-RT pulmonary function test, range (median) & \\
\hline Tumor location $^{\mathrm{b}}$ & & & 0.97 & $\mathrm{FEV}_{1}(\%)$ & $30-124(72.5)$ \\
\hline LLL & $15(15)$ & $10(66.7)$ & & DLCO (\%) & $23-125(64)$ \\
\hline
\end{tabular}

LUL $\quad 25(25) \quad 15(60)$

RLL $\quad 9(9) \quad 6(66.7)$

RML $6(6) \quad 4(66.7)$

RUL $\quad 45(45) \quad 24(53.3)$

\section{Tumor histology}

$\begin{array}{lll}\text { Adenocarcinoma } & 57(57) & 30(52.6) \\ \text { Neuroendocrine } & 1(1) & 1(100) \\ \text { Non-small } & 18(18) & 13(72.2) \\ \text { Squamous } & 24(24) & 15(62.5)\end{array}$

\section{Treatment type ${ }^{b}$}

$\begin{array}{lll}\text { IMRT } & 64(64) & 35(54.7) \\ \text { Proton } & 13(13) & 9(69.2) \\ \text { 3D Conformal } & 23(23) & 15(65.2)\end{array}$

\section{Chemotherapy status}

$\begin{array}{lll}\text { Concurrent } & 86(86) & 52(60.5) \\ \text { RT alone } & 14(14) & 7(50) \\ \text { Smoking history } & & \\ \text { Currently } & 28(28) & 15(53.6) \\ \text { Former } & 66(66) & 40(60.6) \\ \text { Never } & 6(6) & 4(66.7)\end{array}$

Interval between staging PET/CT and start of RT

Median (range) in days 18 (3-69) 15 (3-69)

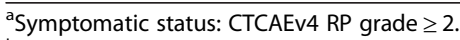

'Yates' continuity correction applied.

IQR: Inter-quartile range.

Note: Hypothesis testing for association used the Mann-Whitney $U$ test for continuous predictors; Pearson's chi-squared test for marginal homogeneity for categorical predictors.

PreRT SUV $\mathbf{S}_{\mathbf{9 5}}, \mathrm{V}_{\mathbf{3 0}}$ and age predict for radiation pneumonitis Age was the only non-modifying factor found to be significantly associated with the development of symptomatic RP using the Mann-Whitney U hypothesis test. Univariate logistic regression analyses are summarized in
Table 2 Treatment characteristics and outcomes

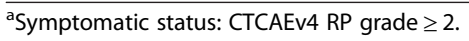

Table 3. Odds of grade $\geq 2$ radiation pneumonitis inas well as $\mathrm{V}_{30}$. SUV $\mathrm{SU}_{95}$ was the most significant independent predictor of post-radiation lung toxicity $(\mathrm{p}<0.0049)$. In addition, significant partial effects were observed for SUV $_{95}(\mathrm{p}<0.0027), \mathrm{V}_{30}(\mathrm{p}<0.007)$, and age $(\mathrm{p}<0.0026)$ in the multiple logistic regression analysis provided in Table 4. For a given age and value of $\mathrm{V}_{30}$, each incremental increase in $\mathrm{SUV}_{95}$ of size 0.1 was associated with a 1.5 -fold increase (95\% CI: $1.1-1.9, \mathrm{p}<0.0027$ ) in the partial odds of symptomatic RP. A patient of average age (64) and $\mathrm{V}_{30}$ (23.8) with a value of $\mathrm{SUV}_{95}=1.2(1.5)$ is 1.4 (6.9) times more likely to develop symptomatic RP when compared to a patient presenting with $\mathrm{SUV}_{95}=1$ (0.5) of the same age and identical $V_{30}$. Additionally, the partial odds of symptomatic RP increased 2.2-fold with each increase in age of 1 year and 1.1-fold with each unit increase in $V_{30}$, respectively.

Receiver Operating Characteristic (ROC) analysis derived from pre-treatment $\mathrm{SUV}_{95}, \mathrm{~V}_{30}$, and age to predict symptomatic (grade $\geq 2$ ) radiation pneumonitis is shown in Figure 3. The area under the ROC curve derived from the multiple logistic regression inference was found to be $0.78(95 \% \mathrm{CI}=0.69-0.87)$ with Youden's optimal sensitivity $=92 \%$ and specificity $=51 \%$. The distribution of symptomatic and asymptomatic is plotted against $\mathrm{SUV}_{95}$ and $\mathrm{V}_{30}$ in Figure 4. Recursive partition analysis for classification of RP symptoms using pre-treatment $\mathrm{SUV}_{95}$ and $\mathrm{V}_{30}$ in 3 cohorts is also shown. The optimal partition (assuming identical misclassification costs) derives from classifying patients with pre-treatment $\mathrm{SUV}_{95}>0.949$ or $\mathrm{V}_{30}>27.14$ as symptomatic, patients with $\mathrm{SUV}_{95}<0.949$ and $\mathrm{V}_{30}<27.14$ as asymptomatic. The joint classification tree results in sensitivity $=98 \%$ and specificity $=37 \%$. creased with $\mathrm{SUV}_{\text {mean }}, \mathrm{SUV}_{\mathrm{SD}}, \mathrm{SUV}_{80}, \mathrm{SUV}_{90}$, and $\mathrm{SUV}_{95}$ 
Table 3 Logistic regression analysis for grade $\geq \mathbf{2}$ RP

\begin{tabular}{|c|c|c|c|c|}
\hline Predictor & Coefficient & SE & Odds ratio $(95 \% \mathrm{Cl})$ & p-value \\
\hline$S U V_{\max }$ & 0.16 & 0.095 & $1.2(0.97,1.4)$ & 0.10 \\
\hline$S U V_{\text {mean }}$ & 0.34 & 0.15 & $1.4(1.1,1.9)$ & $<0.02^{\mathrm{a}, \mathrm{b}}$ \\
\hline$S U V_{S D}^{1}$ & 1.5 & 0.54 & $4.4(1.5,12.8)$ & $<0.0057^{\mathrm{a}, \mathrm{b}}$ \\
\hline SUV 18 & 0.31 & 0.13 & $1.4(1.1,1.7)$ & $<0.013^{\mathrm{a}, \mathrm{b}}$ \\
\hline $\mathrm{SUV}_{90}^{1}$ & 0.33 & 0.12 & $1.4(1.1,1.7)$ & $<0.0061^{\mathrm{a}, \mathrm{b}}$ \\
\hline SUV $_{95}^{1}$ & 0.33 & 0.12 & $1.4(1.1,1.7)$ & $<0.0049^{\mathrm{a}, \mathrm{b}}$ \\
\hline$H U_{\max }^{2}$ & 0.013 & 0.02 & $1(0.97,1.1)$ & 0.51 \\
\hline$H U_{\text {mean }}^{2}$ & -0.09 & 0.34 & $0.91(0.47,1.8)$ & 0.79 \\
\hline$H U_{s d}^{2}$ & -0.18 & 0.93 & $0.83(0.14,5.1)$ & 0.85 \\
\hline $\mathrm{HU}_{80}^{2}$ & -0.07 & 0.37 & $0.93(0.46,1.9)$ & 0.85 \\
\hline $\mathrm{HU}_{90}^{2}$ & -0.10 & 0.32 & $0.91(0.48,1.7)$ & 0.76 \\
\hline $\mathrm{HU}_{95}^{2}$ & -0.10 & 0.32 & $0.91(0.48,1.7)$ & 0.76 \\
\hline$M^{\prime} D^{3}$ & 0.88 & 0.58 & $2.4(0.78,7.5)$ & 0.13 \\
\hline$V_{5}^{3}$ & 0.15 & 0.53 & $1.2(0.41,3.3)$ & 0.77 \\
\hline$V_{10}^{3}$ & 0.58 & 0.55 & $1.8(0.6,5.3)$ & 0.30 \\
\hline$V_{20}^{3}$ & 1.1 & 0.63 & $3(0.87,10.5)$ & 0.081 \\
\hline$V_{30}^{3}$ & 1.2 & 0.57 & $3.3(1.1,10.3)$ & 0.035 \\
\hline FEV1 $(\%)^{2}$ & -0.13 & 1 & $0.88(0.12,6.6)$ & 0.897 \\
\hline $\operatorname{DLCO}(\%)^{2}$ & -1.2 & 1.1 & $0.31(0.034,2.9)$ & 0.31 \\
\hline
\end{tabular}

${ }^{1}$ Scaled by 10.

${ }^{2}$ Scaled by 0.01 .

${ }^{3}$ Log-transformation applied to improve model fit.

${ }^{\text {a}}$ Sequentially rejective Bonferroni method applied to adjust for multiplicity at the $\alpha=0.05$ familywise significance level.

${ }^{b}$ Odds of grade $\geq 2$ radiation pneumonitis increased with $S_{U V} V_{\text {mean, }} S_{S V_{S D}}$

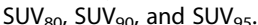

Note: $\mathrm{SE}=$ standard error of the estimated coefficient parameter; $\mathrm{Cl}=$ confidence interval for the odds ratio; $p$-values derived from two-sided hypothesis tests using Wald chi-square.

\section{SUV $_{95}$ Influences Time to Development of Radiation} Pneumonitis

Among patients who developed symptomatic RP, the average time from start of RT to symptomatic development was observed to be 3.5 months for patients with $\mathrm{SUV}_{95}>0.99$

Table 4 Multiple logistic regression analysis for grade $\geq \mathbf{2}$ $\operatorname{RP}(\mathbf{N}=100)$

\begin{tabular}{lllll}
\hline Predictor & Coefficient & SE & Odds ratio $(\mathbf{9 5 \% ~ C l})$ & p-value \\
\hline Intercept & -6.1 & 1.7 & & \\
SUV $_{95}^{\mathrm{a}}$ & 0.40 & 0.13 & $1.5(1.1-1.9)$ & $<\mathbf{0 . 0 0 2 7}$ \\
Age $^{\mathrm{b}}$ & 0.79 & 0.26 & $2.2(1.3-3.7)$ & $<0.0026$ \\
$\mathrm{~V}_{30}$ & 0.09 & 0.034 & $1.1(1-1.2)$ & $<\mathbf{0 . 0 0 7}$ \\
\hline
\end{tabular}

${ }^{\mathrm{a} S c a l e d}$ by 10 .

${ }^{\mathrm{b}}$ Standardized age was used with origin corresponding to the mean of 64 . Note: $\mathrm{SE}=$ standard error of the estimated coefficient parameter; $\mathrm{Cl}=$ confidence interval for the odds ratio; Stepwise backward model selection based on Akaike information criterion was used; Symptomatic radiation pneumonitis was conditionally independent of tumor location, stage, histology, smoking status, $\mathrm{MLD}$, and RT modality in the presence of $\mathrm{SUV}_{95}, \mathrm{~V}_{30}$, and age; $\mathrm{p}$-values derived from two-sided hypothesis tests using Wald chi-square; significant partial effects suggest that the odds of symptomatic radiation pneumonitis increased with $\mathrm{SUV}_{95}, \mathrm{~V}_{30}$, and age; Nagelkerke coefficient of multiple determination $\mathrm{R}^{2}=0.32$.

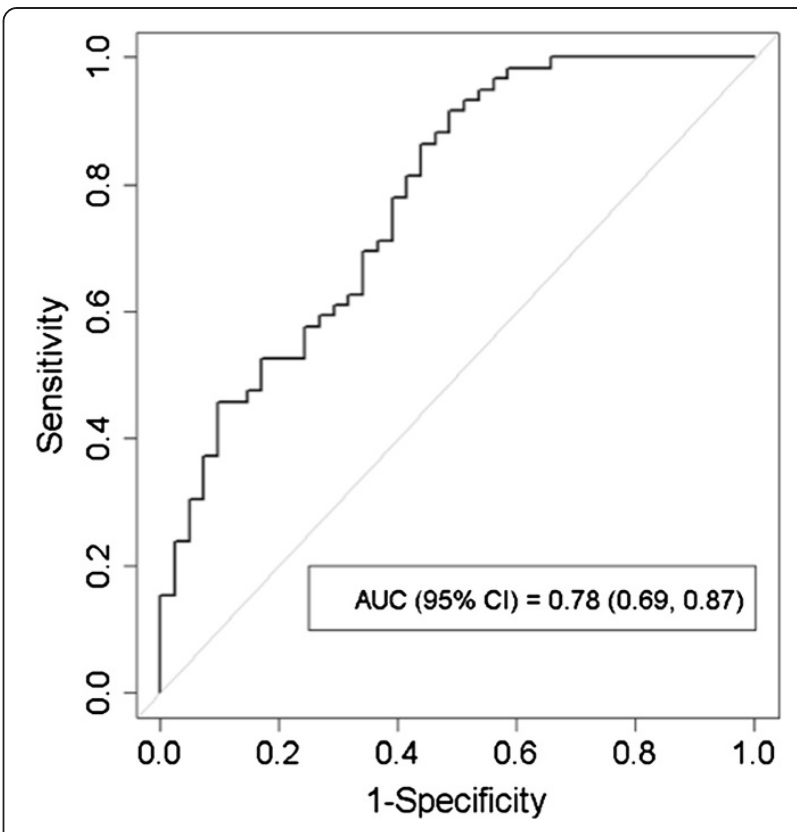

Figure 3 Receiver operating characteristics curve for RP symptoms. Receiver Operating Characteristic (ROC) curve (solid) derived from pre-treatment $S U V_{95}, V_{30}$, and age to predict symptomatic (grade $\geq 2$ ) radiation pneumonitis. The area under the ROC curve derived from the multiple logistic regression inference was found to be 0.78 (95\% Cl: 0.69 - 0.87) with Youden's optimal sensitivity $=92 \%$ and specificity $=51 \%$.

and 4.5 months for patients with $\mathrm{SUV}_{95}<0.99$. KaplanMeier curves were constructed to compare time to radiation pneumonitis symptoms among subsets of patients within observed terciles of $\mathrm{SUV}_{95}\left(\mathrm{SUV}_{95}<0.99\right.$, $0.99 \leq \mathrm{SUV}_{95}<1.2, \quad \mathrm{SUV}_{95} \geq 1.2$ ). Figure 5 shows that patients with $\mathrm{SUV}_{95} \geq 1.2$ developed symptoms at a rate $2.39(1.19,4.82)$ times the rate of patients with $\mathrm{SUV}_{95}<0.99$, while patients with $0.99 \leq \mathrm{SUV}_{95}<1.2$ developed symptoms at a rate $2.25(1.12,4.52)$ times greater.

Additionally, multiple Cox proportional hazards regression was used to evaluate the association between $\mathrm{SUV}_{95}$ and time to development of symptomatic RP, adjusted for age and $\mathrm{V}_{30}$ (Table 5). The odds of developing symptomatic RP within a given duration of time increased with $\mathrm{SUV}_{95}$, age, and $\mathrm{V}_{30}$. SUV 95 contributed the most significant partial effect $(p<0.002)$. Given age and $V_{30}$, each incremental 0.1 increase in $\mathrm{SUV}_{95}$ was associated with a 1.2 -fold increase $(1.1,1.3)$ in the partial hazard rate of RP symptom development.

\section{Inter-reviewer agreement for acquisition of $\mathrm{SUV}_{\mathbf{9 5}}$}

Inter-reviewer agreement among three independent reviewers for determination of $\mathrm{SUV}_{95}$ using a representative $10 \%$ of all cases (10 subsampled patients) is plotted in Figure 6. Inter-reviewer deviation was within approximately $6 \%$ of the reviewer average at the $\alpha=0.05$ significance level. 

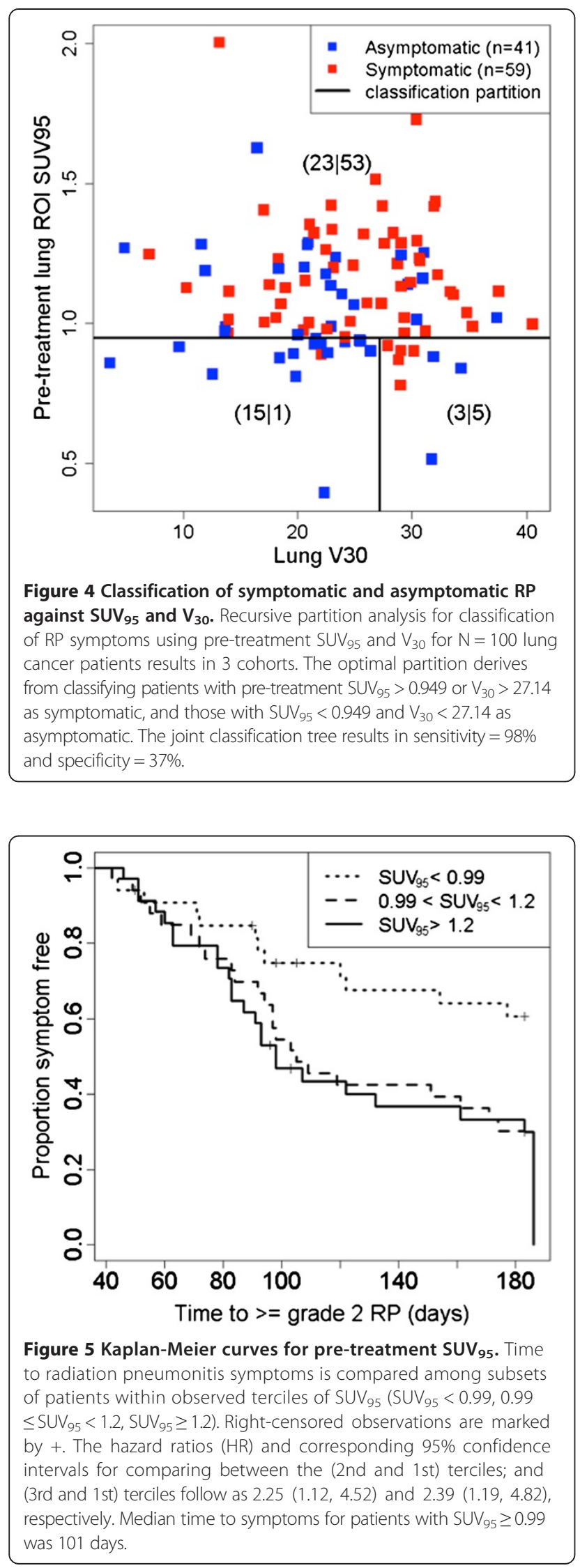

Table 5 Cox proportional hazards regression analysis for time to radiation pneumonitis symptoms $(\mathrm{N}=100)$

\begin{tabular}{lllll}
\hline Predictor & Coefficient & SE & Hazard ratio $(\mathbf{9 5 \%}$ Cl) & p-value \\
\hline SUV $_{95}^{a}$ & 0.18 & 0.057 & $1.2(1.1-1.3)$ & $<\mathbf{0 . 0 0 2}$ \\
Age $^{b}$ & 0.34 & 0.14 & $1.4(1.1-1.8)$ & $\mathbf{0 . 0 1 3}$ \\
$V_{30}$ & 0.05 & 0.02 & $1.1(1-1.1)$ & $\mathbf{0 . 0 1 1}$
\end{tabular}

${ }^{\text {aScaled by } 10 .}$

${ }^{\mathrm{b}}$ Standardized age was used with origin corresponding to the mean of 64 . Note: $\mathrm{SE}=$ standard error of the estimated coefficient parameter; $\mathrm{Cl}=$ confidence interval for the hazard ratio; Stepwise backward model selection based on generalized Akaike information criterion was used; symptomatic radiation pneumonitis was conditionally independent of tumor location, stage, histology, smoking status, treatment type, and MLD in the presence of $S_{U V} V_{95}, V_{30}$, and age; $\mathrm{p}$-values derived from two-sided hypothesis tests using Wald chi-square; the rate of symptom development was increased significantly with $\mathrm{SUV}_{95}, \mathrm{~V}_{30}$, and age.

The observed variation among reviewers reflects the inherent subjectivity associated with the manual intervention to remove PET spill-over activity artifacts (Figure 1) and SUV cold spot artifacts at the lung/diaphragm interface due to respiration. While deviation on the order of $6 \%$ is not innocuous given the magnitude of association between the risk of RP and the pre-treatment $\mathrm{SUV}_{95}$, this represents the $95 \%$ limit of agreement based upon a subset of 10 patients. Thus we expect on average that inter-reader deviation would be on the order of $\pm 3 \%$, which corresponds to only a 0.88 to 1.12 -fold change in the odds of symptomatic RP.

\section{Discussion}

In this study, we demonstrated the potential of a quantitative image derived prognostic biomarker, the $\mathrm{SUV}_{95}$, for the pre-treatment identification of NSCLC patients at high

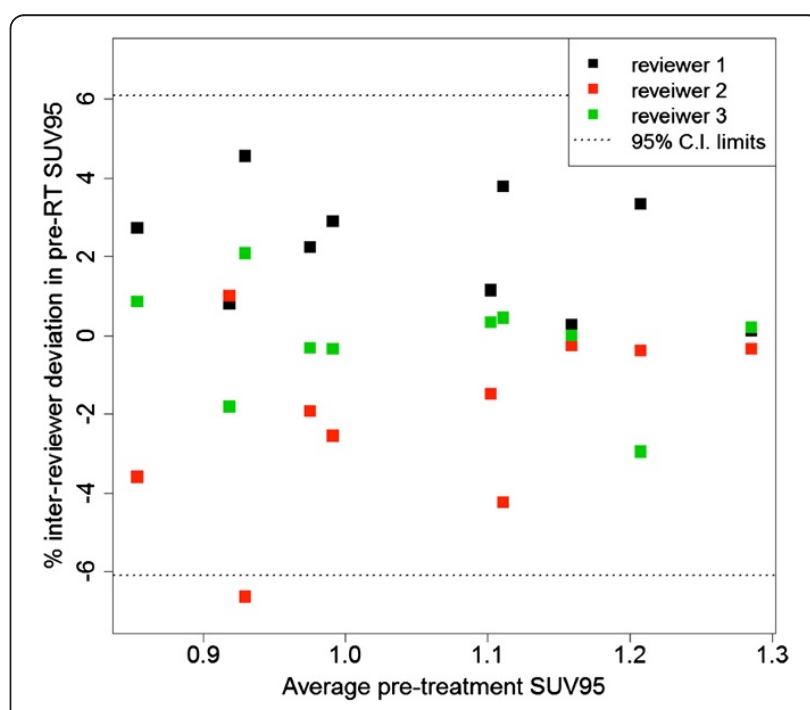

Figure 6 Bland-Altman plot for inter-reviewer agreement in the determination of pre-treatment lung $\mathbf{S U V}_{\mathbf{9 5}}$. Observed and expected percentage deviation from mean $\mathrm{SUV}_{95}$ in a subsample of 10 patients assessed by three independent reviewers. One-way mixed effects ANOVA obtains 95\% confidence boundaries $= \pm 6.10 \%$. 
risk to develop symptomatic RP. This biomarker provides a quantitative assessment of pre-existing pulmonary inflammation [22,39], which in turn predicts the individual subject's ability to tolerate thoracic radiation without toxicity. This study, which includes a mixture of proton and photon treated lung cancer cases, replicates the finding of Petit et al. [24] who studied a photon-only treated NSCLC cohort. Dehing et al. [40] previously analyzed data from a photon-only treated cohort of 438 patients with NSCLC or SCLC to assess predictive value of patient characteristics and dosimetric parameters associated with dyspnea following thoracic chemo-radiotherapy. Univariate models with $\mathrm{V}_{20}$ (mean: $21 \%$, SD: 7.3\%) or MLD (mean: $13.5 \mathrm{~Gy}$, SD: 4.5 Gy) both yielded AUC of 0.47 . The final multivariate model, which included WHO-performance status, smoking status, forced expiratory volume, age, and MLD, yielded an AUC of 0.62 (95\% CI: 0.55-0.69). However, the authors cite that baseline dyspnea scores were not available to rule out the possibility that patients with low FEV1 values already had an elevated dyspnea score prior to treatment. The current study supports the previous findings by Dehing et al. that a combination of patient-related factors and dosimetric parameters, namely the $\mathrm{SUV}_{95}$, $\mathrm{V}_{30}$, and age, is better suited as a prognostic indicator for symptomatic outcomes following thoracic radiotherapy. Pretreatment FDG PET/CT imaging is already routinely obtained for staging of NSCLC [41-43] and has an emerging role in target delineation for radiotherapy treatment planning for NSCLC $[44,45]$. The $\mathrm{SUV}_{95}$, computed from imaging studies already obtained for staging and treatment planning, can be used to stratify toxicity risk without incurring additional cost.

Notably, the significant association between Hounsfield Unit derived parameters and increase in dyspnea reported by Petit et al. [24] did not hold in the current analysis. The difference may arise due to the difference in CT acquisition methods between studies. Although Petit et al. describe both respiratory gated 4D-CT and low-dose CT with intravenous contrast for each patient, it is not clear which CT image set was utilized to calculate the lung region of interest (ROI) Hounsfield Unit values. In this study, Hounsfield Unit ROI parameters were derived utilizing the radiotherapy treatment planning $\mathrm{CT}$, which was a mix of either free-breathing CT (FB-CT) or 4D-CT.

Other imaging modalities have been utilized to estimate the pretreatment symptomatic RP risk. The relationship between the radiation dose distribution and subsequent RP has been well studied and is summarized nicely by Rodrigues et al. [6]. Single photon emission computed tomography (SPECT) perfusion imaging has been utilized to demonstrate radiation-induced lung toxicity $[46,47]$, showing a nearly linear loss of perfusion with radiation dose. Kocak et al. [12] prospectively tested RP prediction models based on pulmonary perfusion and radiation dose distributions using models built from one data set and tested on two other data sets. Those models were unable to segregate patients into high and low risk of RP groups in the test data sets. Others have utilized pretreatment ventilation imaging to predict RP in single cohort retrospective studies [48]; however the ROC AUC was small. Hope et al. [49] developed a 3-parameter model (from the tumor superior-inferior relative position, maximum dose, and dose to the hottest $35 \%$ of the lung volume), which was tested using a separate data set (RTOG 9311) by Bradley et al. [50] and performed poorly. The STRIPE meta-analysis of pneumonitis after chemoradiotherapy for lung cancer [7] found that concurrent paclitaxel, age, and $\mathrm{V}_{20}$ were significant predictive factors with odds ratios of $5.58,1.38$, and 1.07 respectively. Paclitaxel is a radiosensitizer of lung tissue $[3,51]$ that can cause pneumonitis even when used alone [52-54]. The $\mathrm{SUV}_{95}$ quantifies pre-existing pulmonary inflammation, the severity of which may reflect the underlying individual propensity toward an inflammatory response.

For lung cancer clinical trials involving thoracic radiation with pulmonary toxicity as an end-point, the $\mathrm{SUV}_{95}$ can be utilized to (1) ensure equally balanced arms or (2) exclude those who appear to have a nearly $100 \%$ certainty of developing symptomatic pulmonary toxicity. An analysis of a prospective clinical trial conducted by the Radiotherapy Oncology Group (RTOG) indicates higher biologically effective doses of radiotherapy are associated with improved outcomes [55]. However, the recently completed prospective study RTOG 0617 found no advantage as well as increased toxicity in the higher dose arm [13]. Biomarkers such as the $\mathrm{SUV}_{95}$ may be used for stratification to enroll only low RP risk study subjects. The $\mathrm{SUV}_{95}$ can also be utilized to identify a subgroup at high risk for the development of RP symptoms for clinical trials studying RP-prevention drugs. A cohort with an expected high incidence of RP would power a drug RP prevention trial using fewer study subjects to measure a reduction in RP toxicity events.

Our study was limited by the retrospective nature of this analysis, which could contain inherent biases that we are not aware of despite our best efforts to control for potential confounders. The 3D-CRT patients were treated in an earlier time period, which may have accounted for increased toxicities with less modern imaging and treatment planning techniques. Additionally, the 3D PET images were not acquired with motion correlation [56], thus contributing to spatial blurring and spill-over activity artifacts that required manual intervention processes to exclude from data analysis. Pneumonitis grade was scored using the medical record rather than standardized questionnaires. A prospective study addressing the pulmonary toxicity should include standardized survey such as the St. George Respiratory Questionnaire [57]. 


\section{Conclusions}

In the present study, patients with high FDG uptake prior to treatment were more likely to develop symptomatic RP. Our findings may be used to identify patients at high risk for radiation-induced lung damage so that interventions can be developed and fatal RP avoided.

\section{Abbreviations}

RP: Radiation pneumonitis; RT: Radiotherapy; NSCLC: Non-small cell lung cancer; CT: Computed tomography; IP: Interstitial pneumonitis; FDG:

${ }^{18}$ F-2-fluoro-deoxyglucose; PET: Positron emission tomography; SUV: Standard uptake value; MLD: Mean lung dose; CGE: ${ }^{60} \mathrm{Co}$ Gray Equivalents; CTCAE v4: National Cancer Institute Common Terminology Criteria for Adverse Events version 4; ROI: Region of interest; HU: Hounsfield Unit; ROC: Receiver operating characteristic; AUC: Area under the curve; RTOG: Radiotherapy oncology group.

\section{Competing interests}

The authors have no commercial or financial interests related to this study to disclose.

\section{Authors' contribution}

RC contributed to study conception and design, data analysis, and drafting of the manuscript. NP, SA, and DM contributed to data analysis and drafting of the manuscript. SC and AO contributed to data acquisition processes and data analysis. ML and EC contributed to the development of data analysis infrastructure, with further contribution to data analysis processes. $\mathrm{BH}$ performed statistical testing and contributed to drafting of the manuscript. TG formulated study conception and design, and contributed to drafting of the manuscript. All authors provided final approval of the manuscript version to be published.

\section{Acknowledgments}

We extend our warmest gratitude to the thoracic radiation oncology faculty, thoracic surgeons, and gastrointestinal medical oncologists at M. D. Anderson whose patients comprised this study. This work was partially funded by the National Institutes of Health through a National Cancer Institute Grant R21CA141833 and through an NIH Director's New Innovator Award DP2OD007044. RC was partially supported by an NIH Research Scientist Development Award K01CA181292.

\section{Author details}

${ }^{1}$ The University of Texas Health Science Center, Houston, TX, USA. ${ }^{2}$ Divisions of Diagnostic Imaging, Houston, TX, USA. ${ }^{3}$ Quantitative Sciences, Houston, TX, USA. ${ }^{4}$ Radiation Oncology, The University of Texas MD Anderson Cancer Center, Houston, TX, USA. ${ }^{5}$ Department of Computational and Applied Mathematics, Rice University, Houston, TX, USA. 'Baylor College of Medicine, Houston, TX, USA. 'Department of Radiation Oncology, Unit 97, The University of Texas M. D. Anderson Cancer Center, 1515 Holcombe Blvd, Houston, TX 77030, USA.

Received: 7 October 2013 Accepted: 2 March 2014

Published: 13 March 2014

\section{References}

1. Ghafoori P, Marks LB, Vujaskovic Z, Kelsey CR: Radiation-induced lung injury. Assessment, management, and prevention. Oncology (Williston Park) 2008, 22(1):37-47. discussion 52-3.

2. Roberts CM, Foulcher E, Zaunders JJ, Bryant DH, Freund J, Cairns D, Penny R, Morgan GW, Breit SN: Radiation pneumonitis: a possible lymphocyte-mediated hypersensitivity reaction. Ann Intern Med 1993, 118(9):696-700.

3. Taghian AG, Assaad SI, Niemierko A, Kuter I, Younger J, Schoenthaler R, Roche M, Powell SN: Risk of pneumonitis in breast cancer patients treated with radiation therapy and combination chemotherapy with paclitaxel. J Natl Cancer Inst 2001, 93(23):1806-11.

4. Hart J, McCurdy MR, Ezhil M, Wei W, Khan M, Luo D, Munden R, Johnson V, Guerrero T: Radiation pneumonitis: Correlation of Toxicity with the Pulmonary Metabolic Radiation Response. Int J Radiat Oncol Biol Phys 2008, 71(4):967-971.
5. McCurdy M, Wazni M, Martinez J, McAleer M, Guerrero T: Exhaled nitric oxide predicts radiation pneumonitis in esophageal and lung cancer patients receiving thoracic radiation. Radiotherapy and Oncology 2011, 101:443-448.

6. Rodrigues G, Lock M, D'Souza D, Yu E, Van Dyk J: Prediction of radiation pneumonitis by dose-volume histogram parameters in lung cancer-a systematic review. Radiotherapy and Oncology 2004 71(2):127-138.

7. Palma D, Senan S, Tsujino K, Barriger R, Rengan R, Moreno M, Bradley J, Kim T, Ramella S, Marks L, de Petris L, Stitt L, Rodrigues G: Predicting radiation pneumonitis after chemoradiation therapy for lung cancer: An international individual patient data meta-analysis. Int. J. Radiation Oncology Biol. Phys. 2013, 85(2):444-450.

8. Allen AM, Czerminska M, Janne PA, Sugarbaker DJ, Bueno R, Harris JR, Court L, Baldini EH: Fatal pneumonitis associated with intensity-modulated radiation therapy for mesothelioma. Int J Radiat Oncol Biol Phys 2006, 65(3):640-5.

9. Kristensen CA, Nottrup TJ, Berthelsen AK, Kjaer-Kristoffersen F, Ravn J, Sorensen $J B$, Engelholm SA: Pulmonary toxicity following IMRT after extrapleural pneumonectomy for malignant pleural mesothelioma. Radiother Oncol 2009, 92(1):96-9.

10. Rice DC, Smythe WR, Liao Z, Guerrero T, Chang JY, McAleer MF, Jeter MD, Correa A, Vaporciyan AA, Liu HH, Komaki R, Forster KM, Stevens CW: Dosedependent pulmonary toxicity after postoperative intensity-modulated radiotherapy for malignant pleural mesothelioma. Int J Radiat Oncol Biol Phys 2007, 69(2):350-7.

11. Wang JY, Chen KY, Wang JT, Chen JH, Lin JW, Wang HC, Lee LN, Yang PC: Outcome and prognostic factors for patients with non-small-cell lung cancer and severe radiation pneumonitis. International Journal of Radiation Oncology, Biology, Physics. 2002, 54(3):735-41.

12. Kocak Z, Borst GR, Zeng J, Zhou S, Hollis DR, Zhang J, Evans ES, Folz RJ, Wong T, Kahn D, Belderbos JS, Lebesque JV, Marks LB: Prospective assessment of dosimetric/physiologic-based models for predicting radiation pneumonitis. Int J Radiat Oncol Biol Phys 2007, 67(1):178-186.

13. Bradley J, Paulus R, Komaki R, Masters G, Forster K, Schild S, Bogart J, Garces Y, Narayan S, Kavadi V, Nedzi L, Michalski J, Johnson D, MacRae R, Curran W, Choy $\mathrm{H}$ : A randomized phase III comparison of standard-dose (60 Gy) versus high-dose (74 Gy) conformal chemoradiotherapy with or without cetuximab for stage III non-small cell lung cancer: Results on radiation dose in RTOG 0617. J Clin Oncol 2013, 31(supp):7501.

14. Graham MV, Purdy JA, Emami B, Harms W, Bosch W, Lockett MA, Perez CA: Clinical dose-volume histogram analysis for pneumonitis after 3D treatment for non-small cell lung cancer (NSCLC). Int J Radiat Oncol Biol Phys 1999, 45(2):323-9.

15. Claude L, Perol D, Ginestet C, Falchero L, Arpin D, Vincent M, Martel I, Hominal S, Cordier JF, Carrie C: A prospective study on radiation pneumonitis following conformal radiation therapy in non-small-cell lung cancer: clinical and dosimetric factors analysis. Radiother Oncol 2004, 71(2):175-81.

16. Rancati T, Ceresoli GL, Gagliardi G, Schipani S, Cattaneo GM: Factors predicting radiation pneumonitis in lung cancer patients: a retrospective study. Radiother Oncol 2003, 67(3):275-83.

17. Guerrero T, Martinez J, McCurdy M, Wolski M, McAleer M: Elevation in exhaled nitric oxide predicts for radiation pneumonitis. Int. J. Radiation Oncology Biol. Phys. 2012, 82(2):981-988.

18. Makimoto T, Tsuchiya S, Hayakawa K, Saitoh R, Mori M: Risk factors for severe radiation pneumonitis in lung cancer. Japanese Journal of Clinical Oncology. 1999, 29(4):192-7.

19. Sanuki N, Ono A, Komatsu E, Kamei N, Akamine S, Yamazaki T, Mizunoe S, Maeda T: Association of computed tomography-detected pulmonary interstitial changes with severe radiation pneumonitis for patients treated with thoracic radiotherapy. J. Radiat. Res. 2012, 53:110-116.

20. Yamashita H, Kobayashi-Shibata S, Terahara A, Okuma K, Haga A, Wakui R, Ohtomo K, Nakagawa K: Prescreening based on the presence of CT-scan abnormalities and biomarkers (KL-6 and SP-D) may reduce severe radiation pneumonitis after stereotactic radiotherapy. Oncology: Radiation; 2010. 5(32)

21. Jones HA, Clark RJ, Rhodes CG, Schofield JB, Krausz T, Haslett C: In vivo measurement of neutrophil activity in experimental lung inflammation. Am J Respir Crit Care Med 1994, 149(6):1635-1639.

22. Chen DL, Rosenbluth DB, Mintun MA, Schuster DP: FDG-PET imaging of pulmonary inflammation in healthy volunteers after airway instillation of endotoxin. J Appl Physiol 2006, 100(5):1602-1609. 
23. Chen DL, Ferkol TW, Mintun MA, Pittman JE, Rosenbluth DB, Schuster DP: Quantifying pulmonary inflammation in cystic fibrosis with positron emission tomography. Am J Respir Crit Care Med 2006, 173(12):1363-1369.

24. Petit $\mathrm{S}$, van Elmpt W, Oberije C, Vegt E, Dingemans A, Lambin P, Dekker A, de Ruysscher D: Fluorodeoxyglucose uptake patterns in lung before radiotherapy identify areas more susceptible to radiation -induced lung toxicity in non-small-cell lung cancer patients. J. Radiation Oncology Biol. Phys 2011, 81(3):698-705.

25. Pan T, Mawlawi O, Nehmeh SA, Erdi YE, Luo D, Liu HH, Castillo R, Mohan R, Liao Z, Macapinlac HA: Attenuation Correction of PET Images with Respiration-Averaged CT Images in PET/CT. J Nucl Med 2005, 46(9):1481-1487.

26. Strauss LG, Conti PS: The applications of PET in clinical oncology. J NuCl Med 1991, 32(4):623-648

27. Liao Z, Liu H, Komaki R: Target delineation for esophageal cancer. Journa of Women's Imaging 2003, 5(4):177-186.

28. Vedam SS, Keall PJ, Kini VR, Mostafavi H, Shukla HP, Mohan R: Acquiring a four-dimensional computed tomography dataset using an external respiratory signal. Phys Med Biol 2003, 48(1):45-62

29. Pan $T$, Lee TY, Rietzel E, Chen GT: 4D-CT imaging of a volume influenced by respiratory motion on multi-slice CT. Med Phys 2004, 31(2):333-40.

30. Osman MM, Cohade C, Nakamoto Y, Wahl RL: Respiratory motion artifacts on PET emission images obtained using $C T$ attenuation correction on PET-CT. European journal of nuclear medicine and molecular imaging 2003, 30(4):603-606.

31. Proschan MWM: Practical guidelines for multiplicity adjustment in clinical trials. Controlled Clinical Trials. 2000, 21:527-539.

32. Akaike $\mathrm{H}$ : A new look at the statistical model identification. IEEE T Automat Contr 1974, 19(6):716-723.

33. Nagelkerke NJD: A note on a general definition of the co efficient of determination. Biometrika 1991, 78:691-692.

34. DeLong ER, DeLong DM, Clarke-Pearson DL: Comparing the areas under two or more correlated receiver operating characteristic curves: a nonparametric approach. Biometrics 1988, 44(3):837-45.

35. Youden WJ: Index for rating diagnostic tests. Cancer 1950, 3(1):32-35

36. Breiman L, Friedman JH, Olshen RA, Stone CJ: Classification and regression trees, Monterey, Calif. U.S.A.: Wadsworth, Inc.; 1984

37. McCulloch C, Searle S, Neuhaus J, Generalized L, Models M, 2nd ed: Hoboken. NJ: Wiley; 2008

38. Bland JM, Altman DG: Statistical methods for assessing agreement between two methods of clinical measurement. Lancet 1986, 1(8476):307-10.

39. de Prost N, Tucci MR, Melo MFV: Assessment of Lung Inflammation With 18 F-FDG PET During Acute Lung Injury. American Journal of Roentgenology 2010, 195(2):292-300.

40. Dehing-Oberije C, De Ruysscher D, van Baardwijk A, Yu S, Rao B, Lambin P: The importance of patient characteristics for the prediction of radiationinduced lung toxicity. Radiotherapy and Oncology 2009, 91:421-426.

41. Ettinger DS, Akerley W, Bepler G, Blum MG, Chang A, Cheney RT, Chirieac LR, D\&apos; Amico TA, Demmy TL, Ganti AKP, Govindan R, Grannis FW Jahan $\mathrm{T}$, Jahanzeb $\mathrm{M}$, Johnson DH, Kessinger A, Komaki R, Kong F-M, Kris MG, Krug LM, Le Q-T, Lennes IT, Martins R, O\&apos; Malley J, Osarogiagbon RU, Otterson GA, Patel JD, Pisters KM, Reckamp K, Riely GJ: Non-small cell lung cancer. Journal of the National Comprehensive Cancer Network. JNCCN 2010, 8(7):740-801.

42. Fischer B, Lassen U, Mortensen J, Larsen S, Loft A, Bertelsen A, Ravn J, Clementsen P, Høgholm A, Larsen K, Rasmussen T, Keiding S, Dirksen A Gerke O, Skov B, Steffensen I, Hansen H, Vilmann P, Jacobsen G, Backer V, Maltbaek N, Pedersen J, Madsen H, Nielsen H, Højgaard L: Preoperative Staging of Lung Cancer with Combined PET-CT. The New England journal of medicine 2009, 361(1):32-39.

43. Silvestri GA, Gould MK, Margolis ML, Tanoue LT, McCrory D, Toloza E, Detterbeck F: Noninvasive Staging of Non-small Cell Lung Cancer: ACCP Evidenced-Based Clinical Practice Guidelines (2nd Edition). Chest 2007, 132(3_suppl):178S-201S.

44. Mac Manus MP, Hicks RJ: The role of positron emission tomography/ computed tomography in radiation therapy planning for patients with lung cancer. Seminars in nuclear medicine 2012, 42(5):308-319.

45. Holloway CL, Robinson D, Murray B, Amanie J, Butts C, Smylie M, Chu K, McEwan AJ, Halperin R, Roa WH: Results of a phase I study to dose escalate using intensity modulated radiotherapy guided by combined PET/CT imaging with induction chemotherapy for patients with non-small cell lung cancer. Radiother Oncol 2004, 73(3):285-7.
46. Marks LB, Munley MT, Spencer DP, Sherouse GW, Bentel GC, Hoppenworth J, Chew M, Jaszczak RJ, Coleman RE, Prosnitz LR: Quantification of radiation-induced regional lung injury with perfusion imaging. Int $J$ Radiat Oncol Biol Phys 1997, 38(2):399-409.

47. Marks LB, Spencer DP, Bentel GC, Ray SK, Sherouse GW, Sontag MR, Coleman RE, Jaszczak RJ, Turkington TG, Tapson V: The utility of SPECT lung perfusion scans in minimizing and assessing the physiologic consequences of thoracic irradiation. Int J Radiat Oncol Biol Phys 1993, 26(4):659-68.

48. Vinogradskiy Y, Castillo R, Castillo E, Tucker S, Zhongxing L, Guerrero T, Martel M: Using 4DCT-Based Ventilation Imaging to Correlate Lung Dose and Function with Clinical Outcomes. J Radiat Oncol Biol Phys 2013. in press.

49. Hope AJ, Lindsay PE, El Naqa I, Alaly JR, Vicic M, Bradley JD, Deasy JO: Modeling radiation pneumonitis risk with clinical, dosimetric, and spatial parameters. Int J Radiat Oncol Biol Phys 2006, 65(1):112-124.

50. Bradley JD, Hope A, Naqa IE, Apte A, Lindsay PE, Bosch W, Matthews J, Sause W, Graham MV, Deasy JO: A nomogram to predict radiation pneumonitis, derived from a combined analysis of RTOG 9311 and institutional data. Int J Radiat Oncol Biol Phys 2007, 69:984-995.

51. McCurdy M, McAleer MF, Wei W, Ezhil M, Johnson V, Khan M, Baker J, Luo D, Ajani J, Guerrero T: Induction and Concurrent Taxanes Enhance both the Pulmonary Metabolic Radiation Response and the Radiation Pneumonitis Response in Patients with Esophagus Cancer. Int J Radia Oncol Biol Phys 2010, 76(3):816-823.

52. Khan A, McNally D, Tutschka PJ, Bilgrami S: Paclitaxel-induced acute bilateral pneumonitis. Ann Pharmacother 1997, 31(12):1471-1474.

53. Wong P, Leung AN, Berry GJ, Atkins KA, Montoya JG, Ruoss SJ, Stockdale FE: Paclitaxel-induced hypersensitivity pneumonitis: Radiographic and CT findings. Am J Roentgenol 2001, 176(3):718-720.

54. Schweitzer VG, Juillard GJF, Bajada CL, Parker RG: Radiation recall dermatitis and pneumonitis in a patient treated with paclitaxel. Cancer 1995, 76(6):1069-1072.

55. Machtay M, Bae K, Movsas B, Paulus R, Gore EM, Komaki R, Albain K, Sause WT, Curran WJ: Higher Biologically Effective Dose of Radiotherapy Is Associated With Improved Outcomes for Locally Advanced Non-Small Cell Lung Carcinoma Treated With Chemoradiation: An Analysis of the Radiation Therapy Oncology Group. International Journal of Radiation Oncology*Biology*Physics 2012, 82(1):425-434.

56. Didierlaurent D, Ribes S, Caselles O, Jaudet C, Cazalet J-M, Batatia H, Courbon F: A new respiratory gating device to improve 4D PET/CT. Medical Physics 2013, 40(3):32501-1-32501-9.

57. Barr JT, Schumacher GE, Freeman S, LeMoine M, Bakst AW, Jones PW: American translation, modification, and validation of the St. George's Respiratory Questionnaire. Clin Ther 2000, 22(9):1121-1145.

doi:10.1186/1748-717X-9-74

Cite this article as: Castillo et al:: Pre-radiotherapy FDG PET predicts radiation pneumonitis in lung cancer. Radiation Oncology 2014 9:74.

\section{Submit your next manuscript to BioMed Central and take full advantage of:}

- Convenient online submission

- Thorough peer review

- No space constraints or color figure charges

- Immediate publication on acceptance

- Inclusion in PubMed, CAS, Scopus and Google Scholar

- Research which is freely available for redistribution 